\title{
INCIDENCE OF TRICHOMONAS VAGINALIS INFECTION AMONG PATIENTS WHO PRESENTED TO A TERTIARY HOSPITAL- A PROSPECTIVE STUDY
}

\author{
Isha Bansal', Sushant Mohite², Vaibhav Mane, V. R. Pawar', Ravi Singh ${ }^{5}$,Vanisha Dhaka 6 \\ ${ }^{1}$ Senior Resident, Department of Pathology, Bharati Vidyapeeth Medical College and Hospital, Sangli. \\ ${ }^{2}$ Senior Resident, Department of Pathology, Bharati Vidyapeeth Medical College and Hospital, Sangli. \\ ${ }^{3}$ Associate Professor, Department of Pathology, Bharati Vidyapeeth Medical College and Hospital, Sangli. \\ ${ }^{4}$ Professor, Department of Pathology, Bharati Vidyapeeth Medical College and Hospital, Sangli. \\ ${ }_{5}^{5}$ Senior Resident, Department of Pathology, Bharati Vidyapeeth Medical College and Hospital, Sangli. \\ ${ }^{6}$ Senior Resident, Department of Pathology, Bharati Vidyapeeth Medical College and Hospital, Sangli.
}

\begin{tabular}{l}
\hline ABSTRACT \\
BACKGROUND \\
Trichomonas vaginalis (TV) is a parasitic protozoan that infects the genitourinary tract and produces the most common curable \\
sexually transmitted disease (STD) in sexually active women. \\
The aim was to study the prevalence of Trichomonas vaginalis infection in patients.
\end{tabular}

\section{MATERIALS AND METHODS}

This was a 6-month prospective study of all the Pap smear specimens received in the department of pathology. The smears were stained with Pap stain and H \& E stain. After microscopy, if the trophozoite forms of T. vaginalis are seen the diagnosis is confirmed. The clinical details were taken from the case reports and requisition slips.

\section{RESULTS}

During this study, total 1650 Pap smears were received in the department of pathology out of which 149 patients were positive for the trophozoites making the prevalence rate in this study as $9.03 \%$ The 30 - to 39-year-old age group had a significantly higher prevalence of infection (36.23 \%; P < 0.05$)$ than the 20 - to 29-year-old $(27.51 \%)$ and 40-to 49-year-old age groups $(20.13 \%$; $\mathrm{P}>$ $0.05)$. The lowest rates of infection were observed in those $<20$ years of age $(6.71 \%)$ and $>50$ years of age $(9.39 \% ; P>0.05)$.

\section{CONCLUSION}

The prevalence of TV was more in sexually active age group and its diagnosis is important as it can cause adverse effects in Antenatal care (ANC) period.

\section{KEYWORDS}

Pap smear, Trichomonas.

HOW TO CITE THIS ARTICLE: Bansal I, Mohite S, Mane V, et al. Incidence of Trichomonas vaginalis infection among patients who presented to a tertiary hospital - A prospective study. J. Evolution Med. Dent. Sci. 2016;5(100):7384-7387, DOI: $10.14260 /$ jemds/2016/1671

\section{BACKGROUND}

Trichomonas vaginalis (TV) is a parasitic protozoan that infects the genitourinary tract and produces the most common curable sexually transmitted disease (STD) in sexually active women in all age groups.[1,2,3,4,] Potential sequelae of this STD in females include pelvic inflammatory disease, ectopic pregnancy, premature labour, tubal factor infertility, adverse pregnancy outcome, reproductive tract infection, and the potential for an increase in the risk of both the transmission and acquisition of human immunodeficiency virus (HIV). $[5,6,7,8]$ Additionally, investigators have reported epidemiologic associations between Trichomonas infection and subsequent cervical neoplasia.[9.10]

Financial or Other, Competing Interest: None.

Submission 10-11-2016, Peer Review 03-12-2016,

Acceptance 10-12-2016, Published 15-12-2016.

Corresponding Author:

Dr. Vaibhav P. Mane,

Flat No.1, Shri Ramshailya Apartment,

Neminathnagar,

Near Ganpati Temple,

Vishrambag, Sangli-416415.

Maharashtra.

E-mail: vaishnailab1060@gmail.com

DOI: $10.14260 /$ jemds $/ 2016 / 1671$

\section{(c) $(1) \risingdotseq$}

Trichomoniasis presents a wide variety of clinical patterns. The spectrum of clinical trichomoniasis in women ranges from the asymptomatic carrier state to vaginitis, with one-third of asymptomatic infected patients becoming symptomatic within 6 months.[1,2,3]

T. vaginalis principally infects the squamous epithelium in the genital tract. The infection, once established, persists for long periods in females but only for a short time in males. It is chiefly a disease of the reproductive years, and rarely are the clinical manifestations of the infection observed before menarche or after menopause. The incubation period is 4 to 28 days in about $50 \%$ of infected individuals. According to the severity of the infection, trichomoniasis may be classified as acute, chronic, or asymptomatic. $[3,4,5$,

The clinical picture in the acute infection reveals diffuse vulvitis due to copious leucorrhoea. The discharge is typically frothy, yellow or green, and mucopurulent. Small punctate haemorrhagic spots may be found on the vaginal and cervical mucosa. $[11,12,13]$ This speckled appearance has been referred to as a "strawberry appearance" and is observed in only $2 \%$ of patients.

In chronic infection, the predominant symptoms are mild, with pruritus and dyspareunia, while the vaginal secretion may be very scanty and mixed with mucus. This form of the disease is particularly important from the epidemiological 
point of view because these individuals are the major source of transmission of the parasite.

Up to 25 to $50 \%$ of infected women are asymptomatic and, $50 \%$ of these women will develop clinical symptoms during the subsequent 6 months.

Although vaginitis is the most common manifestation of $\mathrm{T}$. vaginalis infection in women, Bartholin's gland is an occasional focus of infection. Other complications associated with trichomoniasis include adnexitis, pyosalpinx, endometritis, infertility, low birth weight, and cervical erosion. Trichomoniasis is also associated with increased HIV transmission. $[1,2,5,6$,

Although T. vaginalis infection is regarded primarily as a disease of women, it also occurs in men. Trichomoniasis in men is largely asymptomatic, and these men are considered to be asymptomatic carriers of T. vaginalis.[6.14,15,] Urogenital trichomoniasis in men may be categorised into three groups: an asymptomatic carrier state, identified by investigation of sexual contacts of infected women; acute trichomoniasis, characterised by profuse purulent urethritis; and mild symptomatic disease, which is clinically indistinguishable from other causes of nongonococcal urethritis.[16,17,18]

Diagnosis of Trichomonas vaginalis can be done by different methods. Wet preparations, culture, staining are routinely used methods. But other serological tests like ELISA, IFA and nowadays molecular based methods can also be used for diagnosis. Studies show that the Pap smear has a sensitivity of $60 \%-70 \%$ in detecting the protozoan in an adequately prepared smear. That's why it is a most routinely used diagnostic tool and also it is cheap and rapid.

\section{Aims and Objectives}

1. To see the incidence of Trichomonas Vaginalis infection in patients who attended the OB/GYN Dept. in our hospital with a chief complaint of white or purulent discharge.

2. To study the demographic features like age, clinical symptoms in patients who are diagnosed as having Trichomonas Vaginalis infection.

\section{MATERIALS AND METHODS}

It is a prospective study conducted in Department of Pathology for 6 months. We studied all the Pap smear specimens received in this period. Cases were analysed in detail regarding complete history, clinical examination and other findings. The smears were studied for the presence of Trophozoite of T. vaginalis and the diagnosis of Trichomonas vaginalis was done.

The percentage of $\mathrm{T}$. vaginalis was found in the cases studied.

\section{RESULTS}

Among the 1650 women, 149 were infected with TV (a prevalence rate of $9.03 \%$ ). The 30 - to 39 -year-old age group had a significantly higher prevalence of infection (36.23\%; P $<0.05)$ than the 20- to 29-year-old (27.51\%) and 40- to 49year-old age groups $(20.13 \%$; $P>0.05)$. The lowest rates of infection were observed in those $<20$ years of age $(6.71 \%)$ and $>50$ years of age $(9.39 \%$; $P>0.05)$.

In the present study, in $\mathrm{T}$. vaginalis infected symptomatic patients, foul smelling purulent vaginal discharge and pruritus were the main symptoms ( $67 \%$ ), followed by dysuria and dyspareunia in $32 \%$ each. On per vaginum examination also, discharge was the most common finding followed by cervical erosions and features of pelvic inflammatory disease (PID). Earlier reports from our centre as well as from other centres also support our findings of discharge being the most important feature.

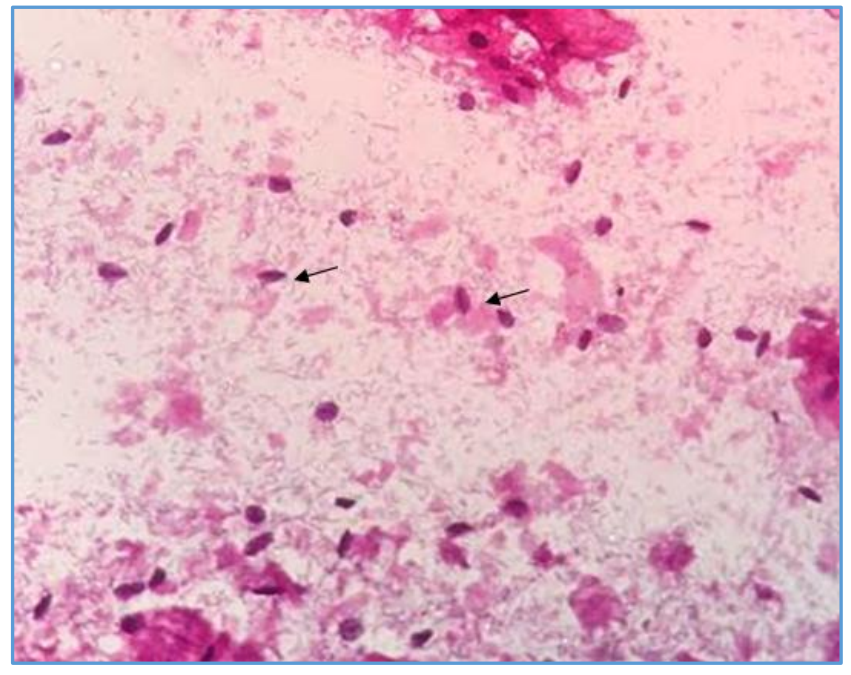

Figure 1. Scattered Trophozoites of Trichomonas Vaginalis (40 X H \& E stain)

\begin{tabular}{|c|c|c|}
\hline Age in Yrs. & Number & Percentage \\
\hline$<20$ & 10 & 6.71 \\
\hline $20-29$ & 41 & 27.51 \\
\hline $30-39$ & 54 & 36.23 \\
\hline $40-49$ & 30 & 20.13 \\
\hline 50 & 14 & 9.39 \\
\hline \multicolumn{2}{|c|}{ Table 1. Age wise Distribution of Cases } \\
\hline
\end{tabular}

\begin{tabular}{|c|c|c|c|}
\hline $\begin{array}{l}\text { Sl. } \\
\text { No. }\end{array}$ & Clinical Presentation & $\begin{array}{c}\text { No. of } \\
\text { Patients }\end{array}$ & Percentage \\
\hline 1 & White discharge & 59 & 39.58 \\
\hline 2 & Yellow discharge & 25 & 16.77 \\
\hline 3 & $\begin{array}{l}\text { Foul smelling dirty } \\
\text { discharge }\end{array}$ & 16 & 10.74 \\
\hline 4 & Itching with discharge & 49 & 32.88 \\
\hline \multirow{2}{*}{\multicolumn{4}{|c|}{\begin{tabular}{|c|c|c|} 
Total & 149 & \\
Tahle ? Clinical Precentation & & \\
\end{tabular}}} \\
\hline & & & ients \\
\hline
\end{tabular}

\section{DISCUSSION}

Trichomonas vaginalis is a parasitic protozoan that is the cause of trichomoniasis, a sexually transmitted disease (STD) of worldwide importance. The disease encompasses a broad range of symptoms ranging from a state of severe inflammation and irritation with a frothy malodorous discharge to a relatively asymptomatic carrier state.

Although cell division has been extensively described using microscopy, the life cycle of T. vaginalis is still poorly understood. Like many other protozoan parasites, it is known to exist only as a trophozoite and lacks a cystic stage. This urogenital pathogen varies in size and shape, with the average length and width being 10 and $7 \mu \mathrm{m}$, respectively. Physiochemical conditions do alter the appearance of the parasite. In culture, the shape of the protozoan tends to be more uniform, i.e., pear shaped or oval, but the parasite takes on a more amoeboid appearance when attached to vaginal epithelial cells. It was thought that these forms are not stages in the life cycle but, rather, that they arise during certain 
unfavourable conditions. However, recent evidence suggests that they may be developmental stages preceding the appearance of mononuclear flagellates.(2) The round forms are morphologically different from the smaller, flagellated ovoid forms.(2) Furthermore, they appear to divide by amitotic budding rather than by the mitotic division of ovoid cells.(1,2) It is not certain how these round forms fit into the development of the organism.

$\mathrm{T}$. vaginalis is an obligate parasite in that it lacks the ability to synthesise many macromolecules de novo, particularly purines, pyrimidines, and many lipids. These nutrients are acquired from the vaginal secretions or through phagocytosis of host and bacterial cells.

Diagnosis of trichomoniasis has traditionally depended on the microscopic observation of motile protozoa in vaginal or cervical secretions, Trichomonads can be differentiated based on their characteristic motility. The sensitivity of this technique varies from as low as $38 \%$ to as high as $82 \%$. Also, the Pap smear examination has its role in the diagnosis. The broth culture using Diamonds medium method is the "gold standard" for the diagnosis of trichomoniasis because it is simple to interpret and requires as few as 300 to 500 trichomonads/mL of inoculum to initiate growth in culture. Direct detection of $\mathrm{T}$. vaginalis antigens in clinical specimens by using monoclonal antibodies holds promise as a rapid method in the diagnosis of trichomoniasis. Recombinant DNA techniques have been increasingly used in clinical laboratories to improve the specificity and sensitivity of $\mathrm{T}$. vaginalis diagnosis. The use of PCR methods helps detect nonviable organisms and also has the ability to detect cells and target sequences in clinical samples that have undergone fixation or partial degradation. PCR assay using the TAV5/TAV6 primer set is a highly sensitive method for the detection of $\mathrm{T}$. vaginalis compared to the culture.

This disease has important medical, social, and economical implications. Women who are infected during pregnancy are predisposed to premature rupture of the placental membranes, premature labour, and low-birth-weight infants. Also linked to this disease are cervical cancer, atypical pelvic inflammatory disease, and infertility.

Increased incidence of $\mathrm{T}$. vaginalis infection is seen in the patients who had their first intercourse at lower age, associated bacterial vaginosis and candidiasis. [19]. Studies in India have shown the prevalence of $\mathrm{T}$. vaginalis infection ranged from $1.2 \%$ to $28.5 \%$ across a variety of populations including obstetrics and gynaecology clinic attendees, STI clinic attendees, commercial sex workers, and communitybased populations.[19] Previous studies estimated that 50$70 \%$ of $\mathrm{T}$. vaginalis infection may be asymptomatic, complicating treatment and prevention efforts.[19] In addition, research suggests that higher number of lifetime sex partners, concurrent infection with other STIs, lower education, and older age are risk factors for $\mathrm{T}$. vaginalis infection.

The total T. vaginalis incidence rate of $9.03 \%$ observed in the present study which was comparable with the Indian study by Purnima Madhivanan, Melissa T. Bartman et al in Mysore. [19] In Nigeria, an overall trichomoniasis prevalence was $2.6 \%$ in women aged 15-64 years[12] while prevalence in Asian studies were as follows: $2.9 \%$ in Chinese women aged $36.3 \pm 6.84$ years, $18.2 \%$ in Palestinian women aged $16-50$ years (2000-2006), $25 \%$ in Turkey and $28.1 \%$ for all sexual transmitted diseases.[14,15,16,17,18]
Trichomoniasis is commonly associated with patients under 25 years of age that are more sexually active than older women. Trichomoniasis was significantly associated $(\mathrm{P}<0.05)$ with being a housewife, belonging to the middle socioeconomic status, and nonuse of contraception.[20] In the present study, the highest prevalence of TV (63\%) was observed in the 20- to 39-year-old age group versus the lowest rate of $6.71 \%$ in the $<20$-year-old age group. Among the sexually active women in the present study, more women in the 30- to 39-year-old age group were infected than in the 20to 29- and 40- to 49- year-old age groups.

Sexually transmitted disease awareness programs, mass media targeted at the younger generation might have contributed to the lower prevalence observed in the 20- to 29year old group. Overall, half of all the women affected were in the 30- to 49-year-old age group.

However, there are other proven and possible methods of spread. Trichomonas can thrive in moist environments; thus, it can survive for up to $45 \mathrm{~min}$. on wet (soiled) clothing, in bath water, and on toilet seats. Thus, although trichomoniasis recognised as a venereal urogenital infection, nonvenereal transmission can occur.

\section{CONCLUSION}

The prevalence of trichomoniasis is more in sexually active age group of female patients, but the overall incidence is decreasing due to increased awareness about the sexually transmitted diseases. But it is important to diagnosis trichomoniasis because it might lead to fatal outcomes in ANC period.

\section{ACKNOWLEDGEMENT}

The authors are thankful to the Department of Obstetrics and Gynaecology, Technicians of Cytology section of Pathology Department, BVDUMC \& H; Sangli for their help and cooperation.

\section{REFERENCES}

1. Schwebke JR, Burgess D. Trichomoniasis. Clin Microbil Rev 2004;17(4):794-803.

2. Garland SM. Trichomonas vaginalis: why we should be screening. Venereology 2001;14(3):116-20.

3. Adu-Sarkodie Y, Opoku BK, Danso KA, et al. Comparison of latex agglutination, wet preparation, and culture for the detection of Trichomonas vaginalis. Sex Transm Infect 2004;80(3):201-3.

4. Moodley P, Wilkinson D, Connolly C, et al. Trichomonas vaginalis is associated with pelvic inflammatory disease in women infected with human immunodeficiency virus. Clin Infect Dis 2002;34(4):519-22.

5. Grice AC. Vaginal infection causing spontaneous rupture of the membranes and premature delivery. Aust $\mathrm{N}$ Z J Obst Gynaecol 1974;14(3):156-8.

6. Wiwanitkit V. Counteraction during movement of spermatozoa by Trichomonas vaginalis observed by visual image analysis: a possible cause of female infertility. Fert Steril 2008;90(3):528-30.

7. Sutton MY, Sternberg M, Nsuami M, et al. Trichomoniasis in pregnant human immunodeficiency virus infected and human immunodeficiency virus-uninfected Congolese 
women: prevalence, risk factors, and association with low birth weight. Am J Obstet Gynecol 1999;181(3):656-62.

8. Brown D. Clinical variability of bacterial vaginosis and trichomoniasis. J Rep Mede 2004;49(10):781-6.

9. Kissinger P, Amedee A, Clark RA, et al. Trichomonas vaginalis treatment reduces vaginal HIV-1 shedding. Sex Transm Dis 2009;36(1):11-6.

10. Viikki M, Pukkala E, Nieminen P, et al. Gynaecological infections as risk determinants of subsequent cervical neoplasia. Acta Oncol 2000;39(1):71-5.

11. Watts DH, Fazzari M, Minkoff $H$, et al. Effects of bacterial vaginosis and other genital infections on the natural history of human papilloma virus infection in HIV-1infected and high-risk HIV-1-uninfected women. J Infect Dis 2005;191(7):1129-39.

12. Abdulazeez A, Alo E, Livingstone R. Epidemiology of urinogenital trichomoniasis in a north-eastern state, Nigeria. The Internet J Parasitic Dis 2007;2(2).

13. Jamali R, Zareikar R, Kazemi A, et al. Diagnosis of Trichomonas vaginalis infection using PCR method compared to culture and wet mount microscopy. Int Med J 2006;5.

14. Bowden FJ, Garnett GP. Trichomonas vaginalis epidemiology: parameterising and analysing a model of treatment interventions. Sex Transm Infect 2000;76(4):248-56.
15. Loo SK, Tang WY, Lo KK. Clinical significance of Trichomonas vaginalis detected in Papanicolaou smear: a survey in female social hygiene clinic. Hong Kong Med J 2009;15(2):90-3.

16. Bakhtiari A, Hajian-Tilaki K, Pasha H. Genital infection by Trichomonas vaginalis in women referring to Babol health centers: prevalence and risk factors. IRCMJ 2008;10(1):16-21.

17. Xueqiang F, Yingzhi Z, Yandfang Y, et al. Prevalence and risk factors of trichomoniasis, bacterial vaginosis, and candidiasis of married women of child-bearing age in rural Shandong. Jpn J Infect Dis 2007;60(5):257-61.

18. Al-Hindi AI, Lubbad AMH. Trichomonas vaginalis infection among Palestinian women: prevalence and trends during 2000-2006. Turk J Med Sci 2006;36(6):371-5.

19. Madhivanan P, Bartman MT, Pasutti L, et al. Prevalence of Trichomonas vaginalis infection among young reproductive age women in India: implications for treatment and prevention. Sex Health 2009;6(4):339-44.

20. Kaur S, Khurana S, Bagga R, et al. Trichomoniasis among women in North India: a hospital based study. Indian J Sex Transm Dis 2008;29(2):76-81. 